\title{
Assessment of gender distribution in dengue surveillance data, the Lao People's Democratic Republic
}

\author{
Nouda Prasith, a Onechanh Keosavanh,, Manilay Phengxay, ${ }^{\mathrm{c}}$ Sara Stone, ${ }^{\mathrm{d}}$ Hannah C Lewis, ${ }^{\mathrm{c}}$ Reiko Tsuyuoka, \\ Tamano Matsui, ${ }^{d}$ Panom Phongmanay, ${ }^{a}$ Bouaphanh Khamphaphongphane ${ }^{b}$ and Yuzo Arimad \\ Correspondence to Yuzo Arima (e-mail: arimay@wpro.who.int).
}

Objective: Adolescent and young adult males account for a large proportion of dengue cases reported through national surveillance systems in the Western Pacific Region. To preliminarily assess the validity of these observed distributions, a field investigation was conducted in the Lao People's Democratic Republic's Savannakhet Province in November 2011.

Methods: Mixed quantitative and qualitative methods were used. Dengue surveillance data from Savannakhet Province, and aggregate hospital admission data from the Savannakhet Provincial Hospital for outpatients and inpatients were analysed by age and sex. Unstructured informal interviews were conducted with local health care workers, primary and secondary school officials and villagers.

Results: An excess of males was found among reported dengue cases in Savannakhet Province in the 15-49 year age group. Females in the same age group, however, were found to access health care more than their male counterparts. Qualitative assessments attributed this distribution to young females being more health-conscious and having greater health care-seeking behaviour.

Discussion: The excess of male dengue cases in the surveillance data appeared to be associated with a truly higher risk of dengue rather than greater health care access or health care-seeking behaviour by young men. This investigation indicated the importance of assessing the reported surveillance data within the context of health care utilization behaviour of the population under surveillance.

n tropical and subtropical Asia Pacific countries, dengue is responsible for considerable public health burden. ${ }^{1,2}$ The mosquito-borne infection can cause severe illness and death and is considered a national priority emerging infectious disease among dengue-endemic countries in the World Health Organization (WHO) Western Pacific Region.

Dengue data reported through national surveillance systems have indicated that adolescent and young adult males are found to be consistently at high risk of dengue. ${ }^{3-5}$ These patterns become apparent when the data are stratified by both age and gender. Studies from the Western Pacific Region have reported that gender differentials in the reported dengue surveillance data may be due to: (1) differentials between the genders in exposure-associated behaviours/ activities $^{5}$ (e.g. working age males exposed to outdoor environments during the day when dengue virus- infected Aedes aegypti mosquitoes are active); (2) biological differences between the sexes (e.g. male-female differences in disease severity once infected); ${ }^{6,7}$ and (3) differential health care access/ seeking behaviour between men and women ${ }^{6,8}$ (e.g. working age males may have better access to health care than their female counterparts or working age females may seek health care less often than their male counterparts).

Males would be the high risk group if the observed male excess in dengue cases was due to differential exposure or to sex differentials in the biological response to infection. However, since the reported case numbers were based on patients captured from health care sites, they may not reflect the true gender distribution of disease risk in the population. ${ }^{3,9,10}$ Health care utilization, if differential by gender, would bias the gender distribution in the surveillance data.

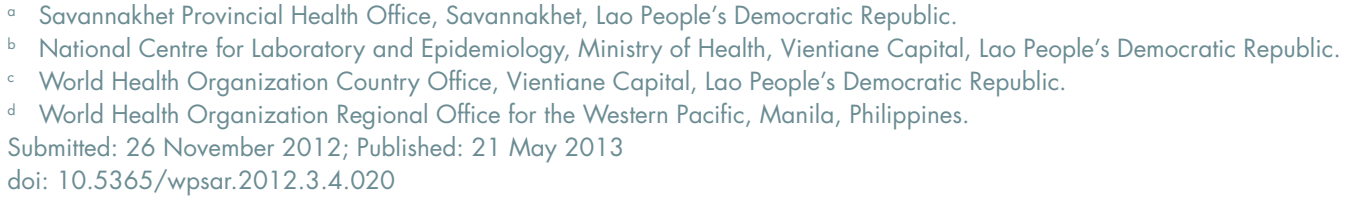


To investigate potential surveillance bias and to assess the validity of the observed dengue distribution, an investigation was conducted in the Lao People's Democratic Republic, a dengue-endemic country in the Region that experienced a dengue epidemic in 2010. The Lao People's Democratic Republic had an estimated population of 6.2 million in $2010^{11}$ and is one of the least populous and least developed countries of South-eastern Asia. ${ }^{12}$ Indicatorbased surveillance is in place for monitoring dengue with weekly reporting of clinically suspected cases presenting at health care facilities; dengue outbreaks are also reported from communities and health facilities through event-based surveillance. This study was conducted in Savannakhet Province, the most populous of the Lao People's Democratic Republic provinces with a population of 824552 persons of which 51\% are female (Figure 1). ${ }^{11}$ We describe both quantitative and qualitative assessments from the dengue surveillance system and from information on health care access and health care-seeking behaviour.

\section{METHODS}

Periurban Savannakhet Province was selected as the province for this assessment for the following reasons: (1) sample size, as the most populous province of the Lao People's Democratic Republic; (2) the existence of a well-functioning provincial health office with access to a reliable surveillance system; and (3) accessibility, transportation and logistical factors. Aggregate dengue surveillance data stratified by age and sex for both the Lao People's Democratic Republic and Savannakhet Province were collected from the National Centre for Laboratory and Epidemiology, Ministry of Health and Savannakhet Provincial Health Office. To calculate notification rates, the latest population data were obtained from the Lao People's Democratic Republic's 2005 census. $^{11}$ Savannakhet provincial-level census data disaggregated by age and sex were not available.

Inpatient and outpatient data from 2010 and 2011 for the top 10 most admitted conditions were collected from Savannakhet Provincial Hospital and stratified by age and sex to assess the gender distribution of health care access. The hospital is a major health care facility for the province, serving all 15 districts and capturing the majority of the reportable diseases and conditions for surveillance purposes. Crude gender comparisons among age groups were made by indicating those age
Figure 1. Location of study site, Savannakhet Province, the Lao People's Democratic Republic

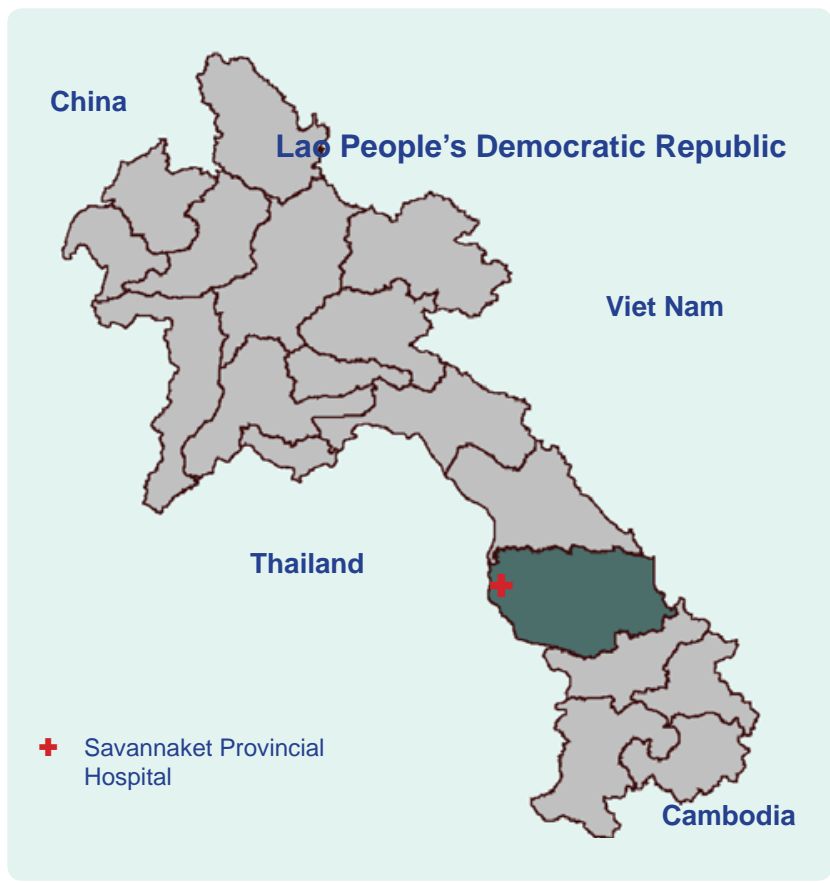

groups that had $20 \%$ or more patients for one gender relative to the other (with at least 10 patients per age and sex category) for each condition. Statistical analyses were done using Stata 11.0 and Microsoft Excel.

Informal, unstructured key informant interviews were conducted among health care workers (Savannakhet Provincial Hospital staff, Savannakhet Provincial Epidemiology Unit staff, Othomphone District Health Office staff, Othomphone District Hospital staff and Phin Tai Health Centre staff) and local community members (Ban Na village health volunteer, Ban Na Lao People's Democratic Republic Women's Union Leader, Ban Na Primary school and Secondary School board and teachers). Othomphone District Hospital covers five health centres in Othomphone District. Phin Tai Health Center serves five local villages in Othomphone District with a mean of approximately 15 patients visiting per day. Interviews consisted of both one-on-one and group discussions. Content analysis of the interviews was conducted with tagging of key concepts. Key questions focused on why specific age and gender groups visited health care facilities more than others.

\section{RESULTS}

In 2010, the Lao People's Democratic Republic had 22912 reported dengue cases and 46 deaths, 
Table 1. Number of reported dengue cases, underlying population and notification rate by sex and age group, the Lao People's Democratic Republic, 2010 ( $n=21$ 119)

\begin{tabular}{lrrcrrr}
\hline $\begin{array}{c}\text { Age group } \\
\text { (years) }\end{array}$ & Cases & Population & $\begin{array}{c}\text { Notification rate } \\
\text { (per 1000) }\end{array}$ & Cases & Population & $\begin{array}{c}\text { Notification rate } \\
\text { (per 1000) }\end{array}$ \\
\hline$<5$ & 622 & 351559 & 1.8 & 624 & 349356 & 1.8 \\
$5-15^{*}$ & 4049 & 770922 & 5.3 & 3176 & 745442 & 4.3 \\
$16-40^{*}$ & 6329 & 1129864 & 4.7 & 4625 & 1152083 & 3.4 \\
$>40^{*}$ & 1000 & 548035 & 0.8 & 694 & 574061 & 0.5 \\
Total $^{*}$ & $\mathbf{1 2 0 0 0}$ & $\mathbf{2 8 0 0 3 8 0}$ & $\mathbf{4 . 3}$ & $\mathbf{9 1 1 9}$ & $\mathbf{2 8 2 0 9 4 2}$ & $\mathbf{3 . 2}$ \\
\hline
\end{tabular}

${ }^{*} P<0.05$ comparing notification rate between males and females, chi-squared test.

Figure 2. Number of reported dengue cases by sex and age group, Savannakhet Province, the Lao People's Democratic Republic, $2010(n=2523)$

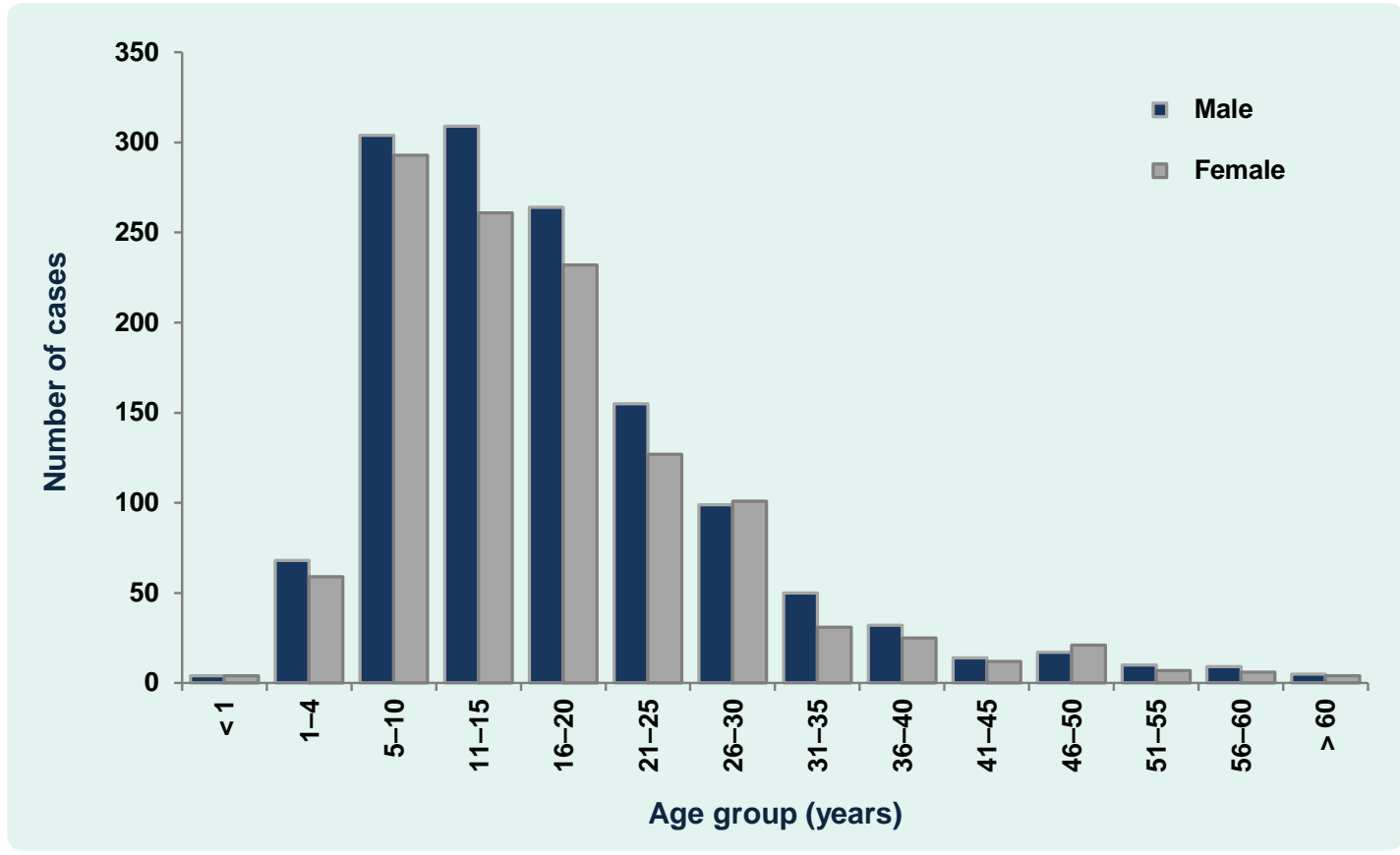

an estimated national annual notification rate of four dengue cases per 1000 persons. This was a significant increase relative to the previous year (7810 cases; chi-squared test, $P<0.01$ ) and the previous two years (2008: 4328 cases; 2007: 5896 cases). Age and sex data were available for 21119 of 22912 cases (92\%). Except for those aged less than five years, males were found to have significantly higher notification rates of dengue than their female counterparts, and the risk difference was greatest among those $16-40$ years of age (Table 1).

Savannakhet Province reported a similarly high annual dengue notification rate of three cases per 1000 persons in 2010 with 2523 reported cases.
The age and sex distribution was similar to that of the overall national pattern with an excess of male cases particularly among those aged 11 to 25 years (Figure 2).

At the Savannakhet Provincial Hospital, similar to the overall provincial and national distributions, an excess of male dengue patients was reported in 2010 and 2011 among 15-49 year olds (Tables 2 and 3). In contrast to 2010, there were only 145 dengue patients reported among inpatients in 2011 , and stratification by age and sex resulted in small numbers.

For 2010 and 2011, the majority of both inpatient and outpatient visits to the provincial hospital were 
Table 2. Ten most common diseases for outpatients by age and sex from Savannakhet Provincial Hospital, Savannakhet Province, the Lao People's Democratic Republic, 2010 and 2011*

\begin{tabular}{|c|c|c|c|c|c|c|}
\hline \multirow{2}{*}{ Diseases } & \multirow{2}{*}{ Sex } & \multicolumn{5}{|c|}{ Age group (years) } \\
\hline & & $<1$ & $1-4$ & $5-14$ & $15-49$ & $>49$ \\
\hline \multicolumn{7}{|l|}{2010} \\
\hline \multirow[t]{2}{*}{ Influenza-like illness } & M & 190 & 644 & 502 & 501 & 84 \\
\hline & $\mathrm{F}$ & 89 & 428 & 382 & 483 & 83 \\
\hline \multirow{2}{*}{ Sore throat } & M & 115 & 647 & 572 & 103 & 13 \\
\hline & $\mathrm{F}$ & 85 & 413 & 421 & 118 & 11 \\
\hline \multirow[t]{2}{*}{ Gastroenteritis } & M & 0 & 2 & 18 & 592 & 217 \\
\hline & $\mathrm{F}$ & 0 & 3 & 15 & 772 & 250 \\
\hline \multirow[t]{2}{*}{ Dengue } & M & 5 & 86 & 344 & 511 & 39 \\
\hline & $\mathrm{F}$ & 2 & 64 & 278 & 449 & 64 \\
\hline \multirow[t]{2}{*}{ Pneumonia } & M & 16 & 81 & 45 & 356 & 114 \\
\hline & $\mathrm{F}$ & 4 & 47 & 25 & 426 & 100 \\
\hline \multirow{2}{*}{ Tuberculosis } & M & 0 & 1 & 2 & 307 & 289 \\
\hline & $\mathrm{F}$ & 0 & 3 & 6 & 354 & 248 \\
\hline \multirow[t]{2}{*}{ Tonsilitis } & M & 6 & 41 & 106 & 267 & 33 \\
\hline & $\mathrm{F}$ & 8 & 31 & 93 & 376 & 41 \\
\hline \multirow[t]{2}{*}{ Bronchitis } & M & 50 & 56 & 54 & 174 & 93 \\
\hline & $\mathrm{F}$ & 31 & 70 & 31 & 193 & 91 \\
\hline \multirow[t]{2}{*}{ Acute watery diarrhoea } & M & 65 & 129 & 62 & 99 & 30 \\
\hline & $\mathrm{F}$ & 31 & 71 & 47 & 107 & 43 \\
\hline \multirow[t]{2}{*}{ Hypertension } & M & 0 & 0 & 0 & 57 & 235 \\
\hline & $\mathrm{F}$ & 0 & 0 & 0 & 44 & 263 \\
\hline \multicolumn{7}{|l|}{2011} \\
\hline \multirow[t]{2}{*}{ Influenza-like illness } & M & 121 & 919 & 357 & 338 & 54 \\
\hline & $\mathrm{F}$ & 61 & 441 & 192 & 472 & 56 \\
\hline \multirow[t]{2}{*}{ Sore throat } & M & 92 & 837 & 372 & 101 & 18 \\
\hline & $\mathrm{F}$ & 37 & 399 & 207 & 148 & 15 \\
\hline \multirow[t]{2}{*}{ Gastroenteritis } & M & 0 & 3 & 25 & 561 & 222 \\
\hline & $\mathrm{F}$ & 0 & 0 & 13 & 882 & 238 \\
\hline \multirow[t]{2}{*}{ Pneumonia } & M & 7 & 65 & 36 & 419 & 112 \\
\hline & $\mathrm{F}$ & 3 & 33 & 25 & 560 & 120 \\
\hline \multirow[t]{2}{*}{ Tonsilitis } & M & 3 & 42 & 81 & 415 & 45 \\
\hline & $\mathrm{F}$ & 1 & 25 & 61 & 586 & 35 \\
\hline \multirow[t]{2}{*}{ Bronchitis } & M & 23 & 86 & 61 & 170 & 128 \\
\hline & $\mathrm{F}$ & 10 & 41 & 21 & 258 & 103 \\
\hline \multirow[t]{2}{*}{ Hypertension } & M & 0 & 0 & 0 & 54 & 444 \\
\hline & $\mathrm{F}$ & 0 & 0 & 0 & 61 & 316 \\
\hline \multirow[t]{2}{*}{ Tuberculosis } & M & 0 & 1 & 20 & 234 & 200 \\
\hline & $\mathrm{F}$ & 0 & 4 & 9 & 204 & 110 \\
\hline \multirow[t]{2}{*}{ Otitis media } & M & 0 & 11 & 22 & 168 & 58 \\
\hline & $\mathrm{F}$ & 0 & 5 & 16 & 268 & 45 \\
\hline Acute watery diarrhoea & M & 14 & 108 & 41 & 95 & 49 \\
\hline & $\mathrm{F}$ & 11 & 56 & 27 & 94 & 40 \\
\hline
\end{tabular}

cells indicate $20 \%$ or more females than males, and cells indicate $20 \%$ or more males than females (with at least 10 cases per age and sex category).

for diseases or conditions with an infectious etiology (Tables 2 and 3). Among outpatients, there was an excess of males among those 14 years or younger and an excess of females among those 15-49 years of age (Table 2). In 2010, 63\% of the 6517 patients younger than 15 years were male, relative to $47 \%$ of the 6289 patients in the $15-49$ year age group $(P<0.01$, chi-squared test); similarly, in $2011,66 \%$ of the 5045 patients less than 15 years old were male, relative to $42 \%$ of the 6088 patients in the $15-49$ year age group $(P<0.01$, chi-squared test). Among inpatients, those 14 years or younger were similarly more likely to be male, but there was no longer an excess of females among those 15-49 years of 
Table 3. Ten most common diseases for inpatients by age and sex from Savannakhet Provincial Hospital, Savannakhet Province, the Lao People's Democratic Republic, 2010 and 2011*

\begin{tabular}{|c|c|c|c|c|c|c|}
\hline \multirow{2}{*}{ Diseases } & \multirow{2}{*}{ Sex } & \multicolumn{5}{|c|}{ Age group (years) } \\
\hline & & $<1$ & $1-4$ & $5-14$ & 15-49 & $>49$ \\
\hline \multicolumn{7}{|l|}{2010} \\
\hline \multirow[t]{2}{*}{ Dengue } & M & 8 & 53 & 425 & 572 & 17 \\
\hline & $\mathrm{F}$ & 5 & 51 & 419 & 443 & 20 \\
\hline \multirow[t]{2}{*}{ Acute watery diarrhoea } & M & 76 & 98 & 22 & 42 & 14 \\
\hline & $\mathrm{F}$ & 35 & 92 & 12 & 49 & 38 \\
\hline \multirow[t]{2}{*}{ Pneumonia } & M & 29 & 130 & 38 & 40 & 38 \\
\hline & $\mathrm{F}$ & 20 & 84 & 29 & 17 & 33 \\
\hline \multirow[t]{2}{*}{ Sore throat } & M & 24 & 74 & 111 & 34 & 6 \\
\hline & $\mathrm{F}$ & 20 & 59 & 86 & 37 & 4 \\
\hline \multirow[t]{2}{*}{ Bronchitis } & M & 112 & 76 & 25 & 5 & 5 \\
\hline & $\mathrm{F}$ & 67 & 52 & 10 & 5 & 7 \\
\hline \multirow[t]{2}{*}{ Gastroenteritis } & M & 0 & 8 & 16 & 73 & 27 \\
\hline & $\mathrm{F}$ & 0 & 4 & 20 & 68 & 51 \\
\hline \multirow[t]{2}{*}{ Hypertension } & M & 0 & 0 & 0 & 25 & 53 \\
\hline & $\mathrm{F}$ & 0 & 2 & 1 & 24 & 72 \\
\hline \multirow[t]{2}{*}{ Typhoid } & M & 0 & 8 & 47 & 34 & 4 \\
\hline & $\mathrm{F}$ & 0 & 1 & 30 & 21 & 14 \\
\hline \multirow[t]{2}{*}{ Tuberculosis } & M & 0 & 0 & 0 & 41 & 48 \\
\hline & $\mathrm{F}$ & 0 & 0 & 0 & 26 & 32 \\
\hline \multirow[t]{2}{*}{ Malaria } & M & 2 & 4 & 18 & 75 & 9 \\
\hline & $\mathrm{F}$ & 1 & 2 & 11 & 23 & 2 \\
\hline \multicolumn{7}{|l|}{2011} \\
\hline \multirow[t]{2}{*}{ Acute watery diarrhoea } & M & 69 & 100 & 15 & 68 & 29 \\
\hline & $\mathrm{F}$ & 34 & 78 & 14 & 52 & 44 \\
\hline \multirow[t]{2}{*}{ Pneumonia } & M & 27 & 118 & 24 & 44 & 61 \\
\hline & $\mathrm{F}$ & 19 & 67 & 20 & 38 & 69 \\
\hline \multirow[t]{2}{*}{ Bronchitis } & M & 109 & 85 & 13 & 8 & 17 \\
\hline & $\mathrm{F}$ & 57 & 45 & 19 & 7 & 12 \\
\hline \multirow[t]{2}{*}{ Gastroenteritis } & M & 1 & 5 & 15 & 82 & 32 \\
\hline & $\mathrm{F}$ & 0 & 7 & 19 & 103 & 48 \\
\hline \multirow[t]{2}{*}{ Sore throat } & M & 17 & 48 & 39 & 31 & 3 \\
\hline & $F$ & 12 & 50 & 28 & 33 & 4 \\
\hline \multirow[t]{2}{*}{ Hypertension } & M & 0 & 0 & 0 & 17 & 69 \\
\hline & $\mathrm{F}$ & 0 & 0 & 0 & 21 & 98 \\
\hline \multirow[t]{2}{*}{ Diabetes } & M & 0 & 0 & 1 & 15 & 45 \\
\hline & $\mathrm{F}$ & 0 & 0 & 1 & 28 & 83 \\
\hline \multirow[t]{2}{*}{ Tuberculosis } & M & 0 & 0 & 2 & 48 & 55 \\
\hline & $\mathrm{F}$ & 0 & 0 & 3 & 29 & 28 \\
\hline \multirow[t]{2}{*}{ Dengue } & M & 0 & 1 & 11 & 52 & 7 \\
\hline & $F$ & 1 & 2 & 15 & 52 & 4 \\
\hline Malaria & M & 0 & 2 & 4 & 72 & 7 \\
\hline & $\mathrm{F}$ & 0 & 1 & 5 & 7 & 4 \\
\hline
\end{tabular}

cells indicate $20 \%$ or more females than males, and cells indicate $20 \%$ or more males than females (with at least 10 cases per age and sex category).

age (Table 3). In 2010, 56\% of 2517 patients less than 15 years old were male, compared to $57 \%$ of 1654 patients in the 15-49 year age group $(P=0.53$, chi-squared test); in 2011, 59\% of 1203 patients less than 15 years old were male, relative to $54 \%$ of 807 patients in the 15-49 year age group $(P=0.03$, chi-squared test). Of those 15-49 years of age, the proportion of males among outpatients (47\% in 2010 and $42 \%$ in 2011 ) as against the inpatients (57\% in 2010 and 54\% in 2011) was significantly different $(P<0.01$ for both years $)$.

Interviews among health care workers indicated that clinicians see more female than male outpatients, 
particularly for milder conditions. Health care staff indicated that many young women of childbearing age were very health conscious and actively sought health care to ensure the best for their newborn. The Phin Tai Health Centre had large posters and banners promoting maternal health care and clinic visits for young women. Interviews conducted at the local Ban Na village with village health volunteers and the Lao Women's Union director found that there has been active promotion for maternal and child health, and women's health had been increasingly emphasized as a priority. It was noted that young adult males prefer pharmacies for self treatment rather than making hospital visits, and some men prefer to send their wives (rather than visiting a clinician themselves) to health care facilities to obtain professional advice and medicines. There was no indication that the excess of male paediatric patients was attributable to better education and thus better access to health care. At the local Ban Na Primary School, 168 of the 286 pupils were female (59\%). While 141 of the 346 pupils were female $(41 \%)$ at the Ban Na Secondary School, the school board members and teachers stated that sick adolescents, whether they are students or not, all go to the same health care facilities as there is no health clinic or special health care privileges through secondary school enrolment. Key informant interviews among local health care providers reported gender differences in exposure-associated behaviours and activities (e.g. playing outdoors, swimming in lakes and being more adventurous).

\section{DISCUSSION}

Similar to other dengue-endemic countries in the Western Pacific Region (e.g. Cambodia, the Philippines and Singapore), ${ }^{3-5}$ adolescent and adult males had significantly higher dengue notification rates than their female counterparts in the Lao People's Democratic Republic overall and in Savannakhet Province. In the Western Pacific Region, gender is a concern given the historical gender norms and its possible association with health care access. ${ }^{10}$ Such concerns raise questions regarding the sex distribution observed in the surveillance data that are not only a reflection of who is getting ill but also who is accessing health care. This investigation highlighted the importance of assessing the surveillance data within the context of health care utilization of the population under surveillance.
This study found that the excess of adolescent and young adult male dengue cases in the surveillance data appears to be associated with a truly higher risk of disease. This male excess was observed despite the backdrop of higher health care-seeking behaviour among their female counterparts. There were more females aged 15-49 years seen as outpatients at the provincial hospital for the top 10 diseases/conditions. This may be due to a truly higher risk of illness among women; however, the opposite pattern was observed among inpatients: a larger number of females aged $15-49$ years were admitted for only two of the 10 diseases/conditions in 2010 and for four in 2011.

Qualitative information supported these findings. Key informant interviews indicated that women of childbearing age are highly health conscious and readily seek health care up to around 35 years of age, as they reportedly want to be healthy during their childbearing years to give birth to healthy children. It was also noted that young adult males prefer self treatment. A recent study found that men of the Lao People's Democratic Republic are significantly more likely to smoke than women, but the proportion of men who sought treatment for respiratory illness was the same between the genders. Men were more likely to answer that they did not think they were sick enough when asked about reasons for not seeking treatment. ${ }^{13}$ Such gender-specific health care-seeking behaviours may explain the excess of females among outpatients but not for more severe inpatients. The presence of a Lao Women's Union leader who championed maternal health and various posters and banners promoting maternal health care and clinic visits for young women were indicative of the current status of health care provision and accessibility in the area. Women's health and family planning campaigns, funded by development agencies, have been actively reaching out to Lao women in recent years. ${ }^{14}$

An excess of male patients for most diseases and conditions was observed among young children. Unlike adolescents and young adults, such excess of young male patients was observed for both outpatients and inpatients with no indication that boys were preferentially being brought to health care. Interviews indicated potential gender differentials in exposureassociated behaviours and activities. In addition, once 
becoming infected (and assuming all other things being equal), there may be biological differences between the sexes such that males may have a more severe outcome to disease relative to their female counterparts. There has been a growing recognition that biological differences between males and females based on genetic, immunological and hormonal factors may determine the susceptibility to disease and clinical outcomes, ${ }^{9,15-18}$ including for dengue infection. ${ }^{6,7}$ Females may mount a more vigorous immune response to infection than males. $^{19}$

Although a greater proportion of outpatients were women and a greater proportion of inpatients were men, there is a concern that women may have not been able to be treated as inpatients due to financial or other reasons. However, such gender bias in health care and treatment opportunities would be expected to result in higher female mortality in the community, which was not observed. Life expectancy for males increased from 50 to 59 years from 1995 to 2005, and the same period saw an increase from 52 to 63 years among females. ${ }^{11}$ While age-stratified sex population data were not available for Savannakhet Province, $51 \%$ of the population were female.

There are several limitations in this study. There was no detailed case-based information available for this investigation. Data were obtained from existing, recorded aggregate summaries, and such information as severity of illness or time from onset to hospital visit was unavailable. Such data would have provided an indication of gender differentials in health care-seeking behaviour and the severity of the condition at the time of health care access. In addition, the number of dengue cases recorded in the provincial hospital was greater than that reported to the national surveillance system of the whole province, possibly because hospital records were based on unverified clinic records (and not strict case definitions). However, such discrepancy is unlikely to be differential by gender, and the sex and age distributions were similar between that reported by the hospital, province and the country. Also, as gender norms, health care-seeking behaviour and access can vary not only between countries but within countries (especially in the Lao People's Democratic Republic among different ethnic groups and urban versus rural subpopulations), ${ }^{10,14,20}$ these findings should not be generalized.
Despite these limitations, the health care access data and the behavioural information show that it is unlikely that the excess of male dengue cases among adolescents and young adults can be explained through higher health care accessibility by young men in Savannakhet Province. It is unlikely that for dengue alone - and not for other diseases or conditions young men would seek health care more than their female counterparts. If anything, when considering the underlying gender-specific health care-seeking behaviour, the number of dengue cases among young men may be underreported. While the specific reasons why this demographic group appears to be at higher risk (i.e. due to exposure and/or biological factors) cannot be determined by this study, the presumed higher risk of dengue for young men agrees with previous findings from other countries in the Region that differ from the Lao People's Democratic Republic in ethnicity, religion, culture and development stage, ${ }^{3}$ such that the observed distributions may have a common risk factor associated with male sex and/or gender-specific behaviours. Adopting a more gender-sensitive approach and taking health care access information into consideration will assist public health practitioners in interpreting the reported surveillance data.

\section{Conflicts of interest}

None declared.

\section{Funding}

None.

\section{Acknowledgements}

The authors would like to express their sincere appreciation to Director Phengta Vongphrachanh and Dr Bounthanom Sengkeopraseuth of the National Centre for Laboratory and Epidemiology, Ministry of Health for providing the data and relevant information; WHO Lao country office: Dr Phanoula Zanarath, for translation and assistance and $\mathrm{Dr}$ Chitsavang Chanthavisouk for sharing knowledge about the Lao context. Lastly, the authors thank the Savannakhet Provincial Health Office and Provincial Hospital, Othomphone District Health Office and District Hospital, Phin Tai Health Center and Ban $\mathrm{Na}$ and Ban Nomvilay, Savannakhet Province. 


\section{References:}

1. Suaya JA et al. Cost of dengue cases in eight countries in the Americas and Asia: a prospective study. The American Journal of Tropical Medicine and Hygiene, 2009, 80:846-855. pmid:19407136

2. Arima $Y$ et al. Epidemiologic Update on the Dengue Situation in the Western Pacific Region, 2011. Western Pacific Surveillance and Response, 2013, 4(2). doi:10.5365/wpsar.2012.3.4.019

3. Anker M, Arima Y. Male-female differences in the number of reported incident dengue fever cases in six Asian countries. Western Pacific Surveillance and Response, 2011, 2(2):17-23. doi:10.5365/wpsar.2011.2.1.002

4. Eong OE. Changing pattern of dengue transmission in Singapore. Dengue Bulletin, 2001, 25:40-44 (http://209.61.208.233/ LinkFiles/Dengue_Bulletin_Volume_25_ch7.pdf, accessed 13 January 2013).

5. Ooi EE, Goh KT, Gubler DJ. Dengue prevention and 35 years of vector control in Singapore. Emerging Infectious Diseases, 2006, 12:887-893. doi:10.3201/10.3201/eid1206.051210 pmid: 16707042

6. Yew YW et al. Seroepidemiology of dengue virus infection among adults in Singapore. Annals of the Academy of Medicine, Singapore, 2009, 38:667-675. pmid:19736569

7. Whitehorn J, Simmons CP. The pathogenesis of dengue. Vaccine, 2011, 29:7221-7228. doi:10.1016/j.vaccine.2011.07.022 pmid:21781999

8. Anders $\mathrm{KL}$ et al. Epidemiological factors associated with dengue shock syndrome and mortality in hospitalized dengue patients in Ho Chi Minh City, Vietnam. The American Journal of Tropical Medicine and Hygiene, 2011, 84:127-134. doi:10.4269/ ajtmh.2011.10-0476 pmid:21212214

9. Skufca $J$ and Arima Y. Sex, gender and emerging infectious disease surveillance: a leptospirosis case study. Western Pacific Surveillance and Response, 2012, 3(3):37-39. doi:10.5365/ wpsar.2012.3.3.001

10. Taking sex and gender into account in emerging infectious disease programmes: an analytical framework. Manila, World Health Organization Regional Office for the Western Pacific, 2011 (http://www.wpro.who.int/topics/gender_issues/Takingsex andgenderintoaccount.pdf, accessed 10 January 2013).
11. Lao population census, 2005. Vientianne, Lao People's Democratic Republic.

12. Western Pacific Country Health Information Profile (CHIPS). 2010 Revision. Manila, World Health Organization Regional Office for the Western Pacific, 2010 (http://www.wpro.who.int/ publications/CHIPS2010.pdf, accessed 9 January 2013).

13. Sychareun $V$ et al. Health seeking behavior assessments for respiratory illness in in the Lao People's Democratic Republic (Laos). Journal of Science, Hue University, 2010, 61:413-425 (http://hueuni.edu.vn/portal/data/doc/tapchi/61_39.pdf, accessed 8 January 2013).

14. Phommavong C, Kingkoe S. National Health Statistic Report, Fiscal Year 2011. Vientiane, Ministry of Health Statistic Division, Department of Planning and Finance, 2012.

15. Institute of Medicine, Committee on Understanding the Biology of Sex and Gender Differences. Exploring the biological contributions to human health. Does sex matter? Washington, DC, National Academy Press, 2001.

16. Ober C, Loisel DA, Gilad Y. Sex-specific genetic architecture of human disease. Nature Reviews. Genetics, 2008, 9:911-922. doi:10.1038/nrg2415 pmid:19002143

17. Department of Economic and Social Affairs. Population Division. Too young to die; Genes or gender? New York, United Nations, 1998.

18. Sex, gender and influenza in achieving gender equality and health equity. Geneva, World Health Organization, 2010 (http:// whqlibdoc.who.int/publications/2010/9789241500111_eng.pdf, accessed 10 November 2012).

19. Klein SL. The effects of hormones on sex differences in infection: from genes to behavior. Neuroscience and Biobehavioral Reviews, 2000, 24:627-638. doi:10.1016/S0149-7634(00)00027-0 pmid:10940438

20. Out-of-pocket spending and health service utilization in Lao P.D.R.: Evidence from the Lao Expenditure and Consumption Surveys. November 2010. Vientiane, The World Bank, 2010 (http://wwwwds.worldbank.org/external/default/WDSContentServer/WDSP/IB /2010/12/22/000356161_20101222022357/Rendered/PDF/ 585790WPOLECS010BOX353808B01PUBLIC1.pdf, accessed 2 January 2013). 
